\title{
Silver diffusion bonding and layer transfer of lithium niobate to silicon
}

\author{
Kenneth Diest, ${ }^{\text {a) }}$ Melissa J. Archer, Jennifer A. Dionne, Young-Bae Park, \\ Matthew J. Czubakowski, and Harry A. Atwater \\ Thomas J. Watson Laboratory of Applied Physics, California Institute of Technology, Pasadena, California \\ 91125, USA
}

(Received 7 July 2008; accepted 8 August 2008; published online 5 September 2008)

\begin{abstract}
A diffusion bonding method has been developed that enables layer transfer of single crystal lithium niobate thin films to silicon substrates. A silver film was deposited onto both the silicon and lithium niobate surfaces prior to bonding, and upon heating, a diffusion bond was formed. Transmission electron microscopy confirms the interface evolution via diffusion bonding which combines interfacial diffusion, power law creep, and growth of (111) silver grains to replace the as-bonded interface by a single polycrystalline silver film. The transferred film composition was the same as bulk lithium niobate. (C) 2008 American Institute of Physics. [DOI: 10.1063/1.2976560]
\end{abstract}

Throughout the past two decades, wafer bonding has become an important electronics fabrication method. ${ }^{1,2}$ The ability to directly bond two materials, with varying lattice constants and crystal orientations, has facilitated the development of a wide range of research, from microelectromechanical systems to silicon-based photonics. Recently, wafer bonding processes have been combined with thin-film layer transfer induced by ion implantation. The combination of these two methods enables thin-film single crystal layer transfer of a wide variety of semiconductors ${ }^{3-5}$ and ferroelectrics. ${ }^{6-8}$ By combining the flexibility of bottom-up processing with the near-ideal optical and electronic properties of single crystal films, these two techniques have become the standard method for producing silicon-on-insulator. $^{4,5}$ In addition, wafer bonding and layer transfer has enabled ultrahigh efficiency, multijunction solar cells to be fabricated by bonding lattice-mismatched semiconductors.

Major challenges with current layer transfer processes concern the thermal mismatch and preparation of the two bonding surfaces. Previous work with lithium niobate and silicon bonding was done using laser-induced forward transfer techniques. ${ }^{8}$ This technique minimized thermal expansion mismatch between the two bonding layers by inducing layer transfer using a carbon dioxide laser rather than traditional thermal cycling. In addition to thermal considerations, extensive work is required to ensure surface planarity, smoothness, and cleanliness of the two surfaces; ${ }^{9-11}$ however, such processes are expensive and inefficient. Further, many of the methods used to produce bondable surfaces can ruin the implantation process and prevent a layer from being transferred. This problem could be circumvented with a reliable method for bonding two roughened or otherwise nonideal surfaces.

In this communication, we report a method for bonding lithium niobate thin films onto silicon substrates. The bond is facilitated through a thin diffusion-bonded silver layer between the lithium niobate and silicon, and the technique allows single crystal thin films of a wide variety of materials to be transferred to virtually any type of substrate. We demonstrate lithium niobate bonding to silicon substrates and elu-

\footnotetext{
a) Author to whom correspondence should be addressed. Electronic mail: diest@caltech.edu.
}

cidate the silver-silver diffusion bonding mechanism. In the context of direct wafer bonding, copper has already been studied for possible applications in three-dimensional electrical interconnects. ${ }^{12,13}$ Our results indicate that silver exhibits a diffusion bonding mechanism similar to that seen for copper. Technologically, a silver bonded interface has the advantage of lower losses for optical device applications. Indeed, silver has been studied extensively for its plasmonic properties in metallodielectric waveguides, resonators, and modulators. $^{14-17}$

Polished, x-cut lithium niobate single crystal samples were obtained from MTI Corporation. These samples were then coimplanted with hydrogen to $6 \times 10^{16}$ ions $/ \mathrm{cm}^{2}$ at 80 $\mathrm{keV}$, followed by a $115 \mathrm{keV}$ helium implantation with a dose of $5 \times 10^{16}$ ions $/ \mathrm{cm}^{2}$. All implantations were done at room temperature. The lithium niobate and silicon (100) substrates were then sequentially sonicated in methanol, acetone, isopropanol, and de-ionized water. Silver was concurrently evaporated on both the implanted lithium niobate and silicon substrates (without removal of the silicon native oxide layer) to a thickness of $400 \mathrm{~nm}$ at $2.5 \AA / \mathrm{s}$. The roughness of the deposited silver was measured using atomic force microscopy, and the contamination of the silver surfaces before bonding was measured using $\mathrm{X}$-ray photoelectron spectroscopy. The silvered surfaces were then bonded together at $500{ }^{\circ} \mathrm{C}$ for $4 \mathrm{~h}$ in a nitrogen environment. The high temperature anneal caused the silver layers to bond together. Simultaneously, the anneal induced crack formation within the lithium niobate at the peak implantation depth. As a result, a layer of lithium niobate, whose thickness corresponds to the peak depth of ion implantation and whose area corresponded to the entire $1 \mathrm{~cm}^{2}$ sample, was transferred to the silicon handle substrate.

Using a focused ion beam, transmission electron microscopy samples were extracted from both the bonded lithium niobate/silver/silicon sample and a silicon (100) substrate with $400 \mathrm{~nm}$ of silver evaporated on it under the same conditions as the bonded sample. Before milling, a $1 \mu \mathrm{m}$ thick platinum layer was locally deposited on the sample to prevent ion-induced lattice damage. Thin sections were extracted from the substrate using focused ion beam patterned microtweezers, as shown in Fig. 1(a). After extraction, the lamellae were thinned to $50 \mathrm{~nm}$ using gallium ions with an accelerating voltage of $30 \mathrm{keV}$. The samples were character- 

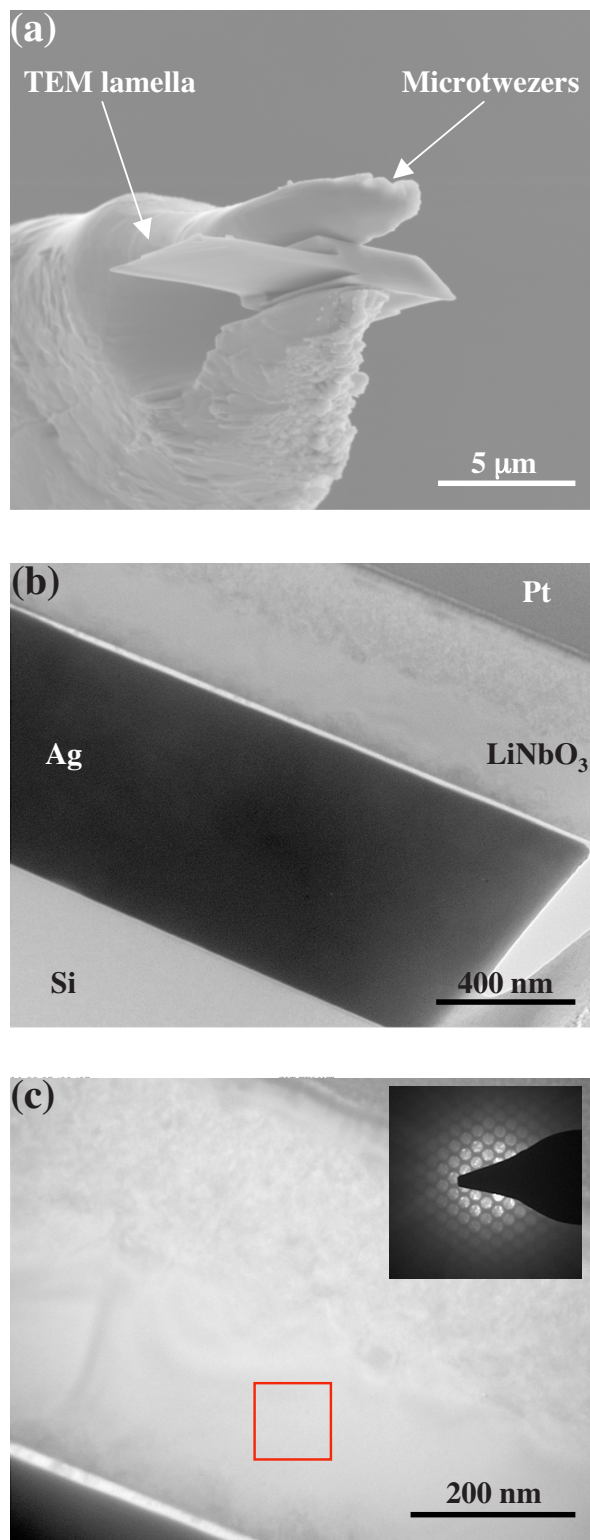

FIG. 1. (Color online) (a) Scanning electron microscope image of the lamella structure that was extracted using the focused ion beam. (b) Transmission electron image of the extracted lamella. From bottom to top the layers are silicon, the bonded silver layer, lithium niobate, and the protective platinum layer. (c) At higher magnification, the lithium niobate layer shows a strained interface, followed by a single crystalline region, and finally an amorphous damage region. The diffraction pattern (inset) was taken in the region enclosed by the red box.

ized using a transmission electron microscope, which was operated at $300 \mathrm{keV}$.

Figure 1(b) shows the lithium niobate sample with the silicon substrate at the bottom, the silver layer as the dark central region, the lithium niobate above it, and the $1 \mu \mathrm{m}$ thick platinum layer in the top right section of the image. The silver layer, which appears as a dark band through the middle of the sample, appears to be continuous from one interface to the other and shows no sign of the original interface between the two silver layers. The lithium niobate layer is shown in detail in Fig. 1(c). From this image, strain contrast from the bonding process can be seen at the interface between the transferred layer and the silver layer (bottom left). This can be attributed to the mismatch in the coefficients of thermal expansion between the silicon, silver, and $\mathrm{x}$-cut lithium niobate layers $\left(4 \times 10^{-6}, 19.1 \times 10^{-6}\right.$, and $5 \times 10^{-6} / \mathrm{K}^{-1}$, respec-
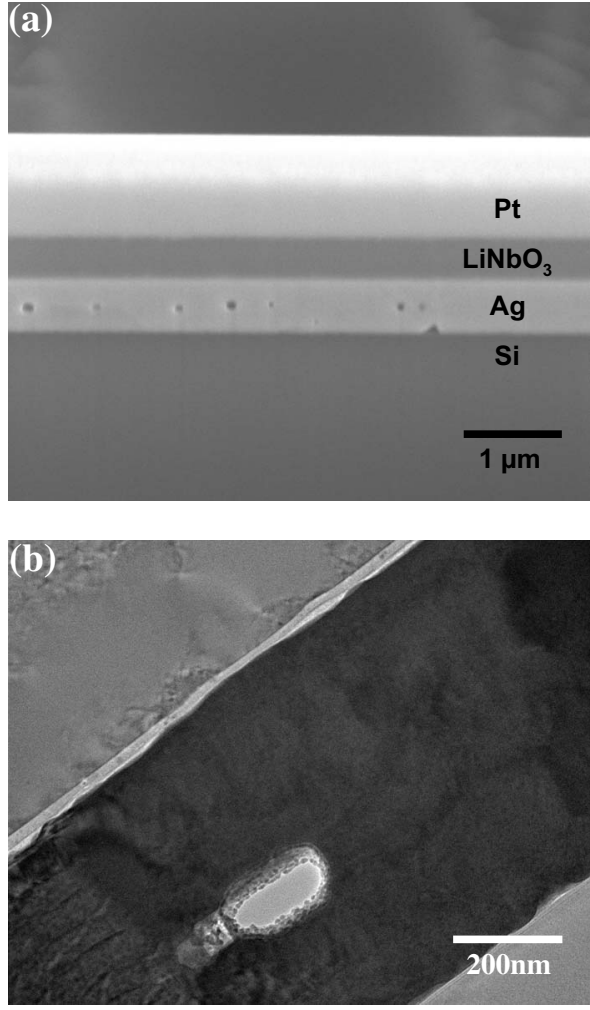

FIG. 2. (a) Scanning electron microscope image of the lithium niobate bonded to silicon with a silver bonding layer. Ellipsoidal voids can be seen half way through the partially bonded silver layer. (b) A transmission electron microscope image of one of the voids shows an intermediate stage between initial contact of the two silver layers and complete bonding, Fig. 3(d).

tively, at room temperature). Through the center of the transferred film, a defect-free single crystal region exists. This is seen in the selected area diffraction pattern shown as an inset, in which the crystal orientation corresponds with the $\mathrm{x}$-cut orientation of the original lithium niobate sample. Above the single crystal region, a region of lattice damage remains as a result of the ion implantation and platinum deposition processes. It has been shown in previous work that a secondary annealing step, after the bonding step, can significantly remove any residual lattice damage from the layer transfer process and return the transferred layer to its original, single crystal state. ${ }^{7}$

X-ray photoelectron spectroscopy confirmed the existence of a thin oxide layer on the surface of the deposited silver films before bonding and atomic force microscopy determined that the root-mean-squared roughness of that surface was $\sim 7.0 \mathrm{~nm}$. These conditions were sufficient to prevent bonding from occurring at room temperature. The samples were initially brought together at room temperature, such that the two silver films only contacted at the asperities of each silver surface. Upon heating to $500{ }^{\circ} \mathrm{C}$, the silver oxide layer became thermodynamically unstable, ${ }^{18,19}$ and diffusion bonding took place. This process, which was previously investigated by Derby and Wallach, ${ }^{20,21}$ consisted of an initial, rapid process of plastic deformation of the asperities of both silver films. Such deformation results in formation of a series of elliptical voids at the fractionally bonded interface. These voids are clearly visible in both scanning electron microscope and transmission electron microscope images, as shown in Figs. 2(a) and 2(b), respectively. 

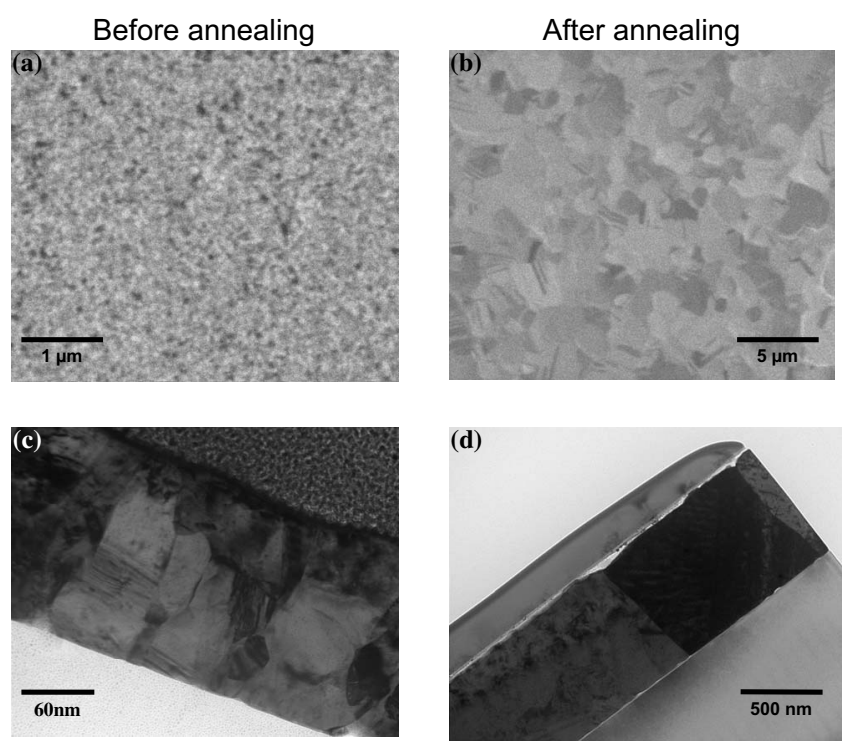

FIG. 3. Focused ion beam images of the deposited silver films before and after annealing, (a) and (b), respectively. The average grain sizes were 53 $\mathrm{nm}$ in (a) and $400 \mathrm{~nm}$ in (b). Transmission electron microscope images of the silver layer before and after bonding, (c) and (d), respectively, show that grain growth during the annealing process has completely removed the interface between the original silver bonding layers.

After the initial stage of plastic deformation, the interfacial voids are removed during the remainder of the bonding process. Voids are removed through a combination of surface diffusion at the void/silver interface, bulk diffusion and grain growth, and power law creep. ${ }^{20,21}$ All three mechanisms contribute to the removal of the bonding interface simultaneously, and the degree to which each mechanism contributes is determined by the bonding material, time, temperature, pressure, and initial surface conditions.

During the bonding process, significant grain growth occurs through the bonding interface, as well as in the plane of the silver film. Focused ion beam images of silver films on $\mathrm{Si}$ before and after annealing show that the average asdeposited grain size was $53 \mathrm{~nm}$, whereas after the annealing the average grain size was $400 \mathrm{~nm}$, as shown in Figs. 3(a) and 3(b), respectively. X-ray diffraction analysis of identical samples (not shown) of the silver films before and after the annealing process show that the grain growth observed is a result of preferential growth of silver (111) grains during the annealing process. Transmission electron microscope images of the silver layer before and after bonding are shown in Figs. 3(c) and 3(d). The sample prior to bonding in Fig. 3(c) exhibits multiple grains whose diameters are small enough that they do not extend throughout the thickness of the film. In contrast, grains extend across the original silver-silver interface after bonding, as shown in Fig. 3(d). Three grains within the silver film extending from the silicon interface to the lithium niobate interface are shown. Note that the inplane grain size in Fig. 3(d) is also significantly larger than in 3(c). As in Fig. 1(b), the interface between the two unbonded silver layers is completely removed after bonding. Also, within each grain the film is single crystalline from the silicon interface to the lithium niobate interface.

After the initial bonding process is complete, if further high temperature processing steps are required, the diffusion bonding process would continue based on the new time, temperature, and pressure conditions. Assuming that the initial heating cycle produces a void-free bond, the bonding would occur through a combination of bulk diffusion and grain growth, along with power law creep. This would further increase the size of the grains that extend from the silicon/ silver interface to the lithium niobate/silver interface.

Finally, we note that the use of this method could generate deep level traps within the silicon substrate. ${ }^{22}$ With a diffusivity on the order of $10^{15} \mathrm{~cm}^{2} / \mathrm{s}$ in silicon, ${ }^{23}$ certain device applications that are dependent on minority carrier lifetimes could be affected. For those devices where silver concentration within the silicon is a concern, a thin diffusion barrier could be deposited between the silver and silicon. ${ }^{24}$ Provided the processing temperature of such a device is kept below $500-600{ }^{\circ} \mathrm{C}$, these layers could be used to prevent adverse silver diffusion.

In conclusion, silver bonding in combination with ion implantation-induced layer transfer has been demonstrated as a method for layer transfer of single crystal lithium niobate onto silicon substrates. Bonding between the two deposited silver layers occurred as a result of diffusion bonding. Scanning electron microscopy and transmission electron microscopy images show that this process completely removed the bonding interface, and within individual grains, the silver was single crystalline from the lithium niobate/silver interface to the silicon/silver interface.

This work was supported by the Army Research Office (ARO-MURI) under Grant No. DAAD 19-01-1-0517 and the Caltech KNI and Materials Science TEM facilities supported by the MRSEC Program of the National Science Foundation under Award No. DMR-0520565.

${ }^{1}$ M. Alexe and U. Gösele, Wafer Bonding: Applications and Technology (Springer, New York, 2004).

${ }^{2}$ Q.-Y. Tong and U. Gösele, Semiconductor Wafer Bonding: Science and Technology (Wiley, New York, 1999).

${ }^{3}$ B. Aspar, M. Bruel, M. Zussy, and A. M. Cartier, Electron. Lett. 32, 1985 (1996).

${ }^{4}$ M. Bruel, Nucl. Instrum. Methods Phys. Res. B 108, 313 (1996).

${ }^{5}$ C. Himcinschi, M. Reiche, R. Scholz, S. H. Christiansen, and U. Gösele, Appl. Phys. Lett. 90, 231909 (2007)

${ }^{6}$ M. Levy, R. M. Osgood, R. Liu, L. E. Cross, G. S. Cargill, A. Kumar, and H. Bakhru, Appl. Phys. Lett. 73, 2293 (1998).

${ }^{7}$ Y.-B. Park, K. Diest, and H. A. Atwater, J. Appl. Phys. 102, 074112 (2007).

${ }^{8}$ Y.-B. Park, B. Min, K. J. Vahala, and H. A. Atwater, Adv. Mater. (Weinheim, Ger.) 18, 1533 (2006).

${ }^{9}$ J. M. Steigerwald, S. P. Murarka, and R. J. Gutmann, Chemical Mechanical Planarization of Microelectronic Materials (Wiley, New York, 1997).

${ }^{10} \mathrm{M}$. Wiegand, M. Reiche, and U. Gösele, J. Electrochem. Soc. 147, 2734 (2000).

${ }^{11} \mathrm{X}$. Zhang and J.-P. Raskin, Electrochem. Solid-State Lett. 7, G172 (2004).

${ }^{12}$ K. N. Chen, A. Fan, C. S. Tan, R. Reif, and C. Y. Wen, Appl. Phys. Lett. 81, 3774 (2002).

${ }^{13}$ K. N. Chen, C. S. Tan, A. Fan, and R. Reif, Electrochem. Solid-State Lett. 7, G14 (2004).

${ }^{14}$ J. A. Dionne, H. J. Lezec, and H. A. Atwater, Nano Lett. 6, 1928 (2006)

${ }^{15}$ J. A. Dionne, L. A. Sweatlock, and H. A. Atwater, Phys. Rev. B 72, 075405 (2005).

${ }^{16}$ L. A. Sweatlock, S. A. Maier, H. A. Atwater, J. J. Penninkhof, and A. Polman, Phys. Rev. B 71, 235408 (2005).

${ }^{17}$ S. I. Bozhevolnyi, V. S. Volkov, E. Devaux, J. Y. Laluet, and T. W. Ebbesen, Nature (London) 440, 508 (2006).

${ }^{18}$ Z. A. Munir, J. Mater. Sci. 14, 2733 (1979).

${ }^{19}$ Z. A. Munir, Powder Metall. 4, 177 (1981).

${ }^{20}$ A. Hill and E. R. Wallach, Acta Metall. 37, 2425 (1989).

${ }^{21}$ B. Derby and E. R. Wallach, Met. Sci. 18, 427 (1984).

${ }^{22}$ R. H. Hopkins and A. Rohatgi, J. Cryst. Growth 75, 67 (1986).

${ }^{23}$ T. C. Nason, G.-R. Yang, K.-H. Park, and T.-M. Lu, J. Appl. Phys. 70, 1392 (1991)

${ }^{24}$ S. Bhagat, H. Han, and T. L. Alford, Thin Solid Films 515, 1998 (2006). 